\title{
Transatlantica
}

Revue d'études américaines. American Studies Journal

\section{L'identité américaine en peinture : American Stories, Paintings of Everyday Life}

$\mathrm{Au}$ Metropolitan Museum of Art, New York, du 12 octobre 2009 au 24 janvier 2010.

Hélène Valance

\section{(2) OpenEdition} Journals

Édition électronique

URL : https://journals.openedition.org/transatlantica/4554

DOI : $10.4000 /$ transatlantica.4554

ISSN : $1765-2766$

Éditeur

Association française d'Etudes Américaines (AFEA)

Référence électronique

Hélène Valance, «L'identité américaine en peinture : American Stories, Paintings of Everyday Life », Transatlantica [En ligne], 2 | 2009, mis en ligne le 01 février 2010, consulté le 04 février 2023. URL http://journals.openedition.org/transatlantica/4554 ; DOI : https://doi.org/10.4000/transatlantica. 4554

Ce document a été généré automatiquement le 4 février 2023.

\section{c) (i) $९$}

Creative Commons - Attribution - Pas d'Utilisation Commerciale - Pas de Modification 4.0 International - CC BY-NC-ND 4.0

https://creativecommons.org/licenses/by-nc-nd/4.0/ 


\section{L'identité américaine en peinture : American Stories, Paintings of Everyday Life}

Au Metropolitan Museum of Art, New York, du 12 octobre 2009 au 24

janvier 2010.

Hélène Valance

1 Exposition intégralement en ligne: http://www.metmuseum.org/special/ americanstories/

\section{Narration et fierté nationale}

2 American Stories: Paintings of Everyday Life offre un véritable panorama de la peinture américaine d'avant 1918: les visiteurs croisent au fil des salles les œuvres les plus célèbres de Copley, Peale, Bingham, Homer, Eakins, Cassatt et Bellows, pour n'en citer que quelques-uns. Pour rassembler un best-of d'une telle envergure, il fallait un thème assez large - stories ici recouvre ce qui pourrait être lu comme une Comédie Humaine en peinture, regroupant scènes de la vie de campagne, de la vie privée, de la vie militaire, etc. Le lien qui unit tous ces tableaux, c'est leur américanité, et l'exposition semble faire pendant à Looking In: Robert Frank's The Americans ${ }^{1}$, au Metropolitan Museum jusque début janvier. Un art américain, qui aspire à l'indépendance, comme le clame dès la première salle The American School ${ }^{2}$ de Matthew Pratt (1765). Plus loin, on retrouve The Gallery of the Louvre ${ }^{3}$ (1831-33) de Samuel Morse : les commissaires de l'exposition semblent ici faire un clin d'œil à l'inventeur, peintre et défenseur ardent de la cause nationale, qui avait voulu dans ce tour de force pictural offrir le meilleur des grands maîtres aux jeunes artistes américains, en leur épargnant les périls, tout autant moraux que financiers, d'un voyage en Europe. La première partie de l'exposition, intitulée «Inventing American Stories » s'appuie sur cette revendication, par la jeune école américaine, d'un statut à la fois artistique et national. En ce sens, le choix de la narration comme fil conducteur, fonctionne à merveille. Petite et grande histoire 
s'entrelacent dans le Paul Revere ${ }^{4}(1768)$ de John Singleton Copley, où la théière d'argent donne à Copley l'occasion de démontrer tout son talent dans le rendu des surfaces et pose le personnage de Revere en artisan d'orfèvrerie, tout en faisant allusion à son rôle dans la vie politique bostonienne : Copley évoque par ce détail la taxation spéciale du thé prévue par les Townsend Acts votés l'année précédente, mesures qui suscitèrent un large mécontentement dans les colonies américaines, et amorcèrent la révolution. L'exposition accompagne ainsi la chronologie nationale, de la conquête de l'Ouest à la Guerre Civile en passant par le conflit mexicain de 1846-48, et jusqu'aux dernières salles le fil narratif tient bon. Un certain nombre de tableaux, d'ailleurs, incluent des scènes de narration, comme The Story of Golden Locks ${ }^{5}$ (1870) de Joseph Seymour Guy ou Reading the Legend ${ }^{6}$ (1852) de Lilly Martin Spencer. Le thème des "histoires ", en revanche, devient plus problématique quand sont abordées les œuvres de Whistler, Chase et Sargent, qui rejetaient justement toute narration. Whistler, qui disait, à propos d'une silhouette noire ajoutée à l'une de ses compositions, qu'il ne se souciait en rien « du passé, du présent, de l'avenir de cette figure noire, posée là parce qu'il fallait du noir à cet endroit ", refusait catégoriquement que sa peinture soit lue en termes religieux, narratifs ou nationalistes, revendiquant un « art pour l'art » ${ }^{7}$.

\section{Une exposition-textbook}

3 American Stories, au contraire, néglige largement les aspects formels des tableaux pour en développer les dimensions sociales, économiques et politiques. Si chaque tableau est accompagné d'un long cartel, l'attention portée aux détails stylistiques reste marginale, les auteurs se limitant au décodage des symboles iconographiques. Dans The Consecration $^{8}$ (George Cochran Lambdin, 1861), la robe grise de l'épouse et l'uniforme bleu de son mari reprennent les couleurs du Nord et du Sud à l'époque de la Guerre Civile, indiquent les commissaires à ceux qui ne l'auraient pas compris. Les perles rouges portées par l'esclave de On to Liberty (Theodor Kaufman, 1867) symbolisent la victoire, le collier bleu de sa compagne, en revanche, est fait d'amulettes protectrices. En présentant des œuvres expressément narratives, «lisibles ", en développant leur analyse sur le mode de l'histoire, cette exposition veut peut-être éviter de prêter le flanc à certaines critiques. A ce titre, il est intéressant d'observer les réactions des visiteurs, selon qu'ils sont Américains, et familiers de ces images devenues de véritables icônes de la culture américaine, ou étrangers, et railleurs devant les insuffisances artistiques qu'ils perçoivent dans de nombreux tableaux ${ }^{10}$. Le projet est aussi sans doute de rendre ces œuvres plus abordables pour le grand public, en créant une exposition didactique, une sorte de manuel scolaire de l'art américain grandeur nature. Les commissaires de l'exposition illustrent ici une longue tradition américaine, et répètent le geste de Charles Wilson Peale invitant les visiteurs à venir s'instruire dans son musée, espace de savoir universel où se croisent œuvres d'art, spécimens botaniques et zoologiques et portraits de grands hommes. De toute évidence, l'exposition s'adresse à un large public, ne serait-ce que par le lieu où elle se tient. Elle accompagne la récente réouverture de la section des arts décoratifs américains au musée, accueillie avec enthousiasme après deux ans de travaux. Elle a sans doute aussi pour but de compenser la fermeture du département de peintures américaines, dont les collections ne seront que partiellement accessibles jusqu'au printemps 2011. Mais cette volonté d'ouverture au plus grand nombre prend également des formes innovantes: toutes les œuvres de l'exposition, ainsi que les textes des cartels, sont accessibles en ligne, et un blog a été 
crée pour l'occasion. Mêlant contextualisation et vulgarisation, les commissaires ont invité des artistes, écrivains, journalistes économiques, et même un critique gastronomique à partager leurs commentaires des œuvres. La lecture des tableaux dans leur contexte historique, exercice si peu commun dans l'histoire de l'art française, est souvent riche, fine, et efficace ${ }^{11}$. On en vient pourtant à regretter que l'entreprise n'ait pas été poussée jusqu'au bout, et que les artefacts d'une culture visuelle plus large n'aient été inclus dans l'exposition, comme le fait le site Picturing America du National Endowment for the Humanities ${ }^{12}$. On aurait pu imaginer, par exemple, de voir aux côtés de The Quilting Frolic ${ }^{13}$ (1813) de John Lewis Krimmel l'un de ces quilts si importants dans la culture visuelle américaine, ou encore un exemple des gravures de Hogarth, cité par le cartel. L'illustration, souvent mentionnée, aurait très bien trouvé sa place dans l'exposition : omniprésente aux Etats-Unis au dix-neuvième siècle, elle fournissait aux artistes des sources d'inspiration et de revenus. De nombreux peintres représentés dans American Stories, dont Winslow Homer, Frederic Remington, John Sloan, ont mené une carrière parallèle d'illustrateur. La séparation entre les "beaux arts » et l'illustration commerciale perdure pourtant ici. Dans la dernière période envisagée, l'on aurait peut-être gagné à mettre en relation la peinture anti-narrative de Whistler et de ses disciples et l'illustration, pour laquelle le tournant du siècle fut un véritable " âge d'or ». D'une certaine manière, le thème de la narration échoue contre cette distinction persistante entre les différents statuts de l'image.

\section{Contre Crystal Bridges ?}

Quant au contexte de cette exposition elle-même, il est difficile de ne pas songer, en voyant rassemblés ces monuments de la peinture américaine, aux débats qui entourent Crystal Bridges, musée d'art américain érigé à Bentonville (Arkansas), par l'héritière de la fortune Wal-Mart ${ }^{14}$. En 2005-06, Alice Walton a suscité l'émotion en jetant son dévolu sur plusieurs œuvres majeures de la peinture américaine, qu'elle entend exposer à Crystal Bridges, créant ex nihilo une collection rivalisant avec les plus grands musées du pays. Les méthodes utilisées ont été tout particulièrement décriées : en privilégiant des oeuvres à statut public mais n'appartenant pas à des musées, en négociant secrètement des sommes faramineuses que des institutions telles que la New York Public Library ou la Jefferson University de Philadelphie ne pouvaient que difficilement refuser, Alice Walton s'est acquis une réputation de mercenaire. Le scandale a eu pour avantage de soulever quelques questions intéressantes, et de mettre à jour les clivages entre domaine public et commercial, entre culture élitiste et populaire, entre l'Amérique rurale et les grandes métropoles du pays. Qu'est-ce qui fait de Kindred Spirits $^{15}$ (Asher Brown Durand, 1849), par exemple, un emblème de la culture américaine, de l' «identité nationale »? A qui ce tableau parle-t-il ? Qu'est-ce qui justifie qu'il soit conservé à New York plutôt qu'à Bentonville? Le sort du chef-d'œuvre de Thomas Eakins, The Gross Clinic ${ }^{16}$ (1875), illustre de nombreux paradoxes : en 2006, Alice Walton propose 68 millions de dollars à la Jefferson University en échange du tableau, qui dort dans une salle auquel le public n'a pas accès. L'université accepte l'offre, mais y ajoute rapidement une condition, digne d'une comédie à l'américaine. Si, dans les semaines qui suivent, la ville de Philadelphie parvient à lever les fonds nécessaires pour égaler l'enchère, The Gross Clinic restera à Philadelphie. Une souscription est lancée, les citoyens de Philadelphie répondent en masse, le tableau est sauvé. Reste que, comme le maire de la ville l'admet lui-même, peu de ceux qui ont participé connaissaient le 
tableau. Comment, dans ce cas, soutenir qu'il est « représentatif » de Philadelphie et de ses habitants, qu'il ne peut quitter la ville? Un conservateur du musée de l'Université de Pennsylvanie mettait en septembre dernier l'événement en parallèle avec une autre anecdote : après la sortie de son film Rocky III, Silvester Stallone proposa d'offrir à la municipalité une statue (sculptée par A. Thomas Schomberg en 1982) qu'il souhaitait voir placée, afin d'immortaliser la silhouette du boxeur, au sommet des marches menant au Philadelphia Museum of Art. L'enthousiasme populaire n'était malheureusement pas partagé par le musée, qui finit par reléguer le bronze à une pelouse voisine, au pied du grand escalier. Qui donc décide des images qui « représentent» le mieux la collectivité ? Par effet de coïncidence, peut-être, American Stories apparaît comme une contre-attaque de la côte Est face à Bentonville. Cependant, si elle s'attache à démontrer les enjeux sociaux, économiques et politiques à l'œuvre dans les tableaux qu'elle présente, l'exposition American Stories n'aborde pas ceux qui occupent l'art américain aujourd'hui. La question du rôle de l'art, et plus généralement $\mathrm{du}$ "patrimoine», dans la vie culturelle, sociale, politique contemporaines reste ouverte.

\section{NOTES}

1.

http://www.metmuseum.org/special/se_event.asp?OccurrenceId=\{1FD57D4D-

FE17-41FA-9025-E2667E36AD27\}

2. http://www.metmuseum.org/special/americanstories/objectView.aspx?oid=1\&sid=2

3. http://www.metmuseum.org/special/americanstories/objectView.aspx? oid=12\&sid=2"

4. http://www.metmuseum.org/special/americanstories/objectView.aspx?sid=2\&oid=2

5. http://www.metmuseum.org/special/americanstories/objectView.aspx?oid=14\&sid=4

6. http://www.metmuseum.org/special/americanstories/objectView.aspx?oid=19\&sid=3

7. James McNeill Whistler, Ten O'Clock, in The Gentle Art of Making Enemies, 1890.

8. http://www.metmuseum.org/special/americanstories/objectView.aspx?oid=3\&sid=4

9. http://www.metmuseum.org/special/americanstories/objectView.aspx?oid=10\&sid=4

10. Voir à ce sujet Loie Fuller, "American Art at the 1889 Paris Exposition: The Paintings They Love to Hate", American Art Vol. 5, No.4 (Automne 1991), pp. 35-53.

11. Elle fait écho à des ouvrages comme Picturing the Nation: Art and Social Change in NineteenthCentury America de David Lubin (Yale University Press, 1994) ou American Genre Painting : The Politics of Everyday Life d'Elizabeth Johns (Yale University Press, 1991).

12. http://picturingamerica.neh.gov/

13. http://www.metmuseum.org/special/americanstories/objectView.aspx?sid=2\&oid=9

14. www.crystalbridges.org/

15. http://www.nga.gov/exhibitions/durandinfo.shtm\#

16. http://www.philamuseum.org/collections/permanent/299524.html 
INDEX

Thèmes : Trans'Arts

\section{AUTEUR}

HÉLĖNE VALANCE

Université Paris 7-Paris-Diderot 